\title{
A personal computer programmable sine-wave generator
}

\author{
BEVERLY MARSHALL-GOODELL, LLOYD FREI, and I. GORMEZANO \\ The University of Iowa, Iowa City, lowa
}

\begin{abstract}
A programmable sine-wave generator has been developed that permits microcomputer control of both discrete and continuous variations in the frequency and amplitude of auditory, visual, or vibrotactile stimuli. The function and design of the sine-wave generator as a peripheral to the Apple II/FIRST system are detailed. Moreover, adaptations of the basic sine-wave circuit are briefly described for interfacing it with other microcomputers (e.g., the IBM PC and compatibles), and for altering the waveform, range, and resolution of the output. Sample programs in Apple II/FIRST and Applesoft BASIC for controlling signal frequency and amplitude are used to illustrate the simplicity of programmable control. The sine-wave generator has many of the capabilities of commercially available ones, at a fraction of the cost.
\end{abstract}

The purpose of the present paper is to report the development of a sine-wave generator capable of providing both between- and within-trial changes in the frequency and intensity of visual, auditory, and vibrotactile stimuli. The function and design of the sine-wave generator as a peripheral device for the Apple II/FIRST system (MarshallGoodell \& Gormezano, 1985; Scandrett \& Gormezano, 1980 ) will be detailed. Adaptations of the basic sine-wave circuit will be briefly described for interfacing with other microcomputers (e.g., IBM PC and compatibles), and for altering the waveform, range, and resolution of the output. Sample programs in Apple II/FIRST and Applesoft BASIC for controlling signal frequency and amplitude will be provided to illustrate the simplicity of programmable control with the sine-wave generator. Lastly, the power and flexibility of the sine-wave generator will be compared to that of more expensive commercial systems.

\section{THE SINE-WAVE GENERATOR}

\section{Functional Characteristics}

The sine-wave generator, under control of the Apple II/ FIRST system, regulates changes in stimulus frequency and amplitude over time, using digital-to-analog (D/A) converters and a voltage-controlled function generator. Separate D/A converters control 256 levels of sine-wave frequency and amplitude, respectively. Selection among the available frequency and amplitude levels is made within a microcomputer control program that sends separate 8 -bit digital codes to each D/A converter. Each time an 8 -bit code is received by the $\mathrm{D} / \mathrm{A}$ converter, the digi-

\footnotetext{
This research was supported by grants from the National Science Foundation and the University of Iowa Foundation (through the generous contributions of H. Gormezano, S. L. Nusser, and W. G. Nusser). Requests for reprints should be sent to I. Gormezano, Department of Psychology, University of Iowa, Iowa City, IA 52242.
}

tal input is translated (within $160 \mathrm{nsec}$ ) to the corresponding output voltage, and the selected output setting is latched until another control code is received. Although it is possible to use D/A converters alone to directly generate complex waveforms (e.g., speech), the addition of a voltage-controlled function generator simplifies the production of standard waveforms by substantially reducing the memory required to store the waveform shape, and freeing the processor to perform other functions such as data collection. The sine-wave generator uses the programmable voltages from the two $D / A$ converters to modulate the frequency and amplitude components of the sine-wave output from a voltage-controlled function generator. As a result, the sine-wave generator provides relatively continuous temporal control of the sine-wave frequency and amplitude. The output from the sine-wave generator can then be amplified and used to drive auditory, visual, or vibrotactile transducers.

\section{Design}

A circuit diagram for the sine-wave generator is shown in Figure 1. The circuit is assembled on a microcomputer prototyping board intended for use in the assembly of unique computer peripherals, using sockets for all integrated circuits and wire-wrap connections between components. The Apple II prototyping board, for example, features a connector strip designed to interface with the Apple II, and a hole pattern suitable for mounting both integrated circuit packages and discrete components (e.g., resistors). Prototyping boards are available from Jameco Electronics and JDR Microdevices ${ }^{1}$ for the IBM PC/XT, $\mathrm{PC} / \mathrm{AT}$, and compatibles that also provide on-board input/ output address decoding, and simplify the programmable control of unique peripheral circuits such as the sinewave generator. In addition, when simultaneous stimulus presentations are required (e.g., dichotic stimulation, masking, and overlapping or simultaneous stimulus compounds), two independent sine-wave generator circuits can 


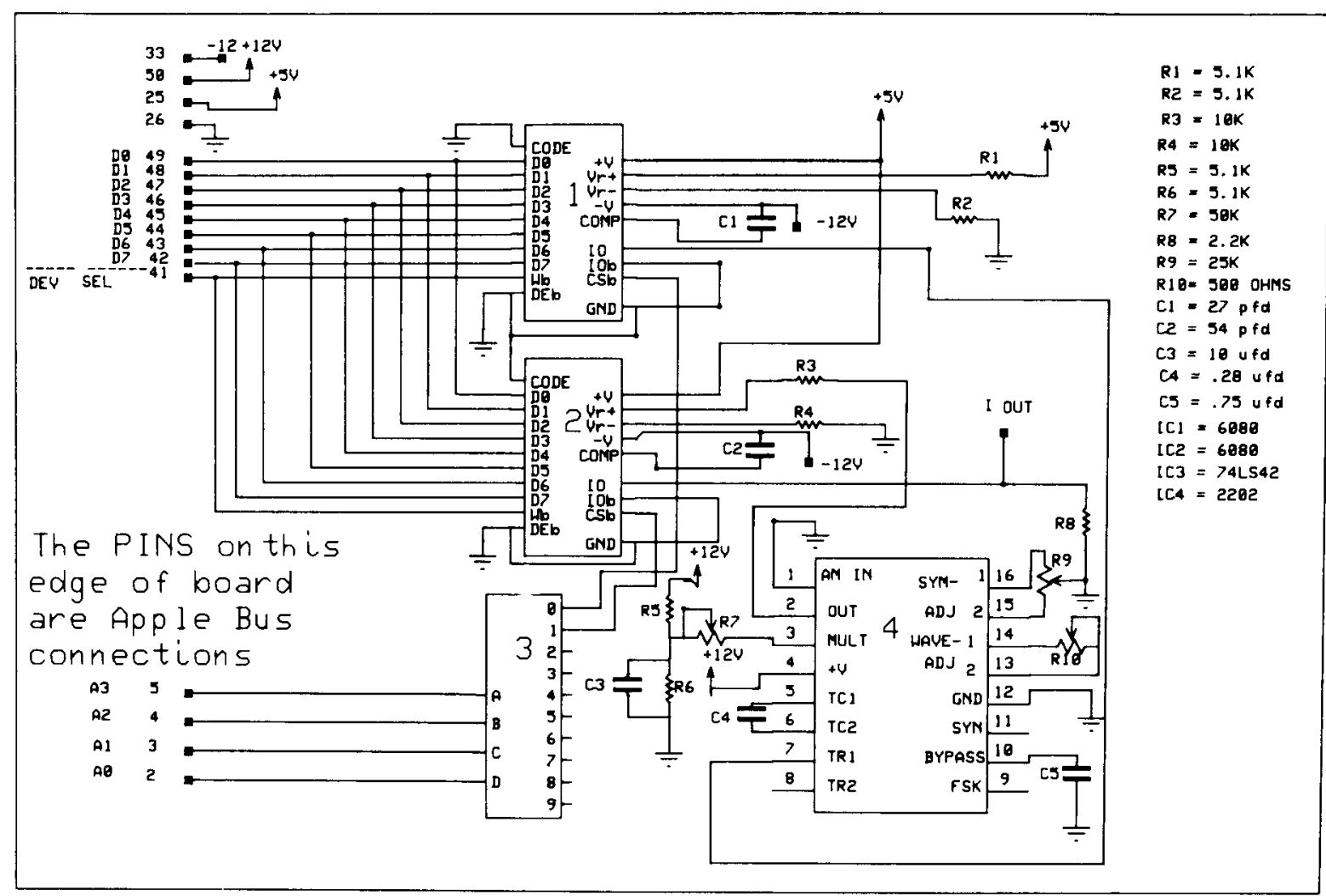

Figure 1. Circuit diagram for the sine-wave generator. The circuit includes four integrated circuits. Numbers 1 and 2 are the D/A converters (AM6080) to control sine-wave frequency and amplitude, respectively. Number 3 is a decoder (7442) used to select which of the D/A converters will receive the digital code from the computer. Number 4 is the function generator (XR-2202).

be wired on a single Apple II prototyping board (four on an IBM-compatible board).

The source of the sine-wave signal is a function generator, XR-2206 (Exar Integrated Systems, 1979), capable of producing sine, square, triangle, ramp, and pulse waveforms of high spectral purity. Although the circuit shown in Figure 1 uses only the sine-wave function, the additional waveform functions are available by selection of different output pins on the function generator. In the present usage, the frequency and amplitude of only the sine-wave output from the function generator is modulated under program control by the external voltages from separate 8-bit high-speed multiplying D/A converters (AM6080, Advanced Micro Devices, 1978). The selection of either the frequency or the amplitude D/A converter is based on the lower 2 bits of the instruction address, and is accomplished by a binary-coded-decimal to decimal decoder (7442). In addition, the sine-wave shaping circuit within the function generator relies on a onetime adjustment of sine-wave shape using component matching to reduce the harmonic content of the output signal from $2.5 \%$ to $0.5 \% .^{2}$

The frequency and amplitude ranges for the sine-wave generator are determined by the value of external timing capacitors, and by timing and gain resistors connected to the function generator. When the sine-wave generator is assembled as described by the circuit in Figure 1, the range of 256 available frequencies is from 17 to $4310 \mathrm{~Hz}$, linearly scaled in approximately $17-\mathrm{Hz}$ steps. The maximum output amplitude of the circuit shown is $1.75 \mathrm{~V}$ (rms), linearly scaled in $6.8-\mathrm{mV}$ steps, ${ }^{3}$ and, therefore, a separate amplifier may be needed to drive the transducer. Moreover, the sine-wave circuit can be adapted to accommodate a range of experimental requirements by changing circuit components, and greater flexibility can be achieved by using hard-wired or programmable switches to select among several component options. Among these adaptations, there are three that are fundamental in determining the system's ability to alter the range and resolution of the sine-wave output. First, the frequency range can be adjusted over the range of the function generator chip $(0.01 \mathrm{~Hz}$ to $1 \mathrm{MHz})$ by changing the timing capacitor across pins 5 and 6 , and adding a timing resistor to pin 7 or 8 . Second, the maximum output amplitude of the function generator can be adjusted in direct proportion to the value of the resistor at pin 3 (i.e., for the sine-wave output, the maximum amplitude is about $60 \mathrm{mV} / \mathrm{k} \Omega$ ). Third, if more than 256 discrete steps are required, the 8-bit D/A converters can be replaced with 12- or 16-bit D/A converters to expand the resolution of frequency and amplitude control. Programmable control of each 12- or 16-bit D/A converter by an Apple II would require two 8-bit addresses and additional decoding circuitry or special 16 -bit write words. 
providing auditory, visual, or vibrotactile stimuli at 256 frequency and amplitude levels; (2) provide continuous temporal control of sine-wave frequency and amplitude; (3) be assembled on a microcomputer peripheral interface board (e.g., Apple II, IBM PC, and compatibles); and (4) be adjusted to alter its waveform, range, and resolution. The very low cost of the sine-wave generator makes it a particularly attractive alternative to commercial systems when multiple programmable signal sources are required.

The diverse output capabilities of a single sine-wave generator make it possible to control a wide variety of stimulus events. First, the sine-wave generator provides an oscillating output that can drive tones, lights, or vibrotactile stimuli. Second, the sine-wave generator can be programmed to produce discrete stimuli at each of 256 frequency and amplitude settings. Third, the sine-wave generator can also be programmed to produce stimulus sequences composed of distinct stimuli selected from the available combinations of frequency and amplitude values.

The within-trial programmable control of changes in stimulus frequency and amplitude provided by the sinewave generator is continuous over time. Thus, the sinewave generator can produce gradual stimulus onsets and offsets, and stimuli that change in frequency and amplitude with the rate of change under program control. Furthermore, both the frequency and amplitude of stimuli can be programmed to follow complex, independent temporal patterns. For example, the frequency of a tone can be programmed to first increase and then decrease, while the amplitude is changed to provide gradual tone onset and offset.

Commercial signal generators that provide programmable control of a signal frequency and amplitude comparable to that of the present sine-wave generator do so using a parallel or serial output port located inside the microcomputer interfaced to a signal source located outside of the computer. In contrast, the sine-wave generator's compact design permits its complete assembly directly on a microcomputer peripheral interface board. Because the sine-wave generator is located completely within the microcomputer cabinet, the need for external cabling between the circuits for signal production and for computer control is eliminated. Moreover, in the present design, the amplitude of the output signal and the step size between amplitude settings are in the millivolt to volt range, and thus no isolation from noise on the microcomputer bus is required. Furthermore, the only shielding from ambient electrical noise that is required is on the single output line to the amplifier. In addition, the simple design of the sine-wave generator circuit can easily be adapted to produce different waveforms, and to alter the range and resolution of the output. In summary, the compact, yet flexible design of the sine-wave generator and the simplicity of its programmable control make it an appealing system relative to more expensive commercial alternatives.

\section{REFERENCES}

Advanced Micro Devices (1978). AM6080 microprocessor system compatible 8-bit high speed multiplying $D / A$ converter. Sunnyvale, CA: Author.

BaLLAS, J. A., \& Houston, J. (1984). Evaluation of a sound-generator chip: The AY3-8910. Behavior Research Methods \& Instrumentation, 16, 282-284.

EXAR INTEGRATED SySTEMS, INC. (1979). Exar function generator data book. Sunnyvale, CA: Author.

Marshall-Goodell, B., \& Gormezano, I. (1985). Apple II/FIRSTCorvus networks. Behavior Research Methods, Instruments, \& Computers, 17, 168-172.

Polich, J., Fischer, A., \& StarR, A. (1983). A programmable multipletone generator. Behavior Research Methods \& Instrumentation, 15, $39-41$.

SCANDRETt, J., \& Gormezano, I. (1980). Microprocessor control and A/D data acquisition in classical conditioning. Behavior Research Methods \& Instrumentation, 12, 120-125.

\section{NOTES}

1. Jameco Electronics, 1355 Shoreway Rd., Belmont, CA 94002; JDR Microdevices, 110 Kenowles Dr., Los Gatos, CA 95030.

2. Waveform adjustment is accomplished by means of monitoring the output signal on an oscilloscope while adjusting two variable resistors that control sine-wave curvature (R10) and symmetry (R9). Specifically, with R9 set to the midpoint, R10 is adjusted for minimum distortion of the sine-wave curvature. Next, R9 is adjusted to further reduce distortions due to asymmetry.

3. A circuit diagram and sample control programs in Apple II/FIRST and Applesoft BASIC for a system that uses a logarithmically scaled D/A converter for programmable tone and white-noise intensity are available from the authors.

(Manuscript received December 14, 1988; revision accepted for publication April 5, 1989.) 